\title{
Polo-like kinase I overexpression is an early event in the progression of papillary carcinoma
}

\section{Y Ito*,', E Miyoshi ${ }^{2}$, N Sasaki ${ }^{3}, K_{\text {Kakudo }}^{5}$, H Yoshida', C Tomoda', T Uruno', Y Takamura', A Miya', K Kobayashi', F Matsuzuka', N Matsuura ${ }^{4}, K_{\text {Kuma' }}$ and A Miyauchi'}

'Kuma Hospital, 8-2-35, Shimoyamate-dori, Chuo-ku, Kobe 650-00II, Japan; ${ }^{2}$ Department of Molecular Biochemistry and Clinical Investigation, Osaka University Graduate School of Medicine, Osaka, Japan; ${ }^{3}$ Department of Biochemistry, School of Allied Health Science, Osaka University Faculty of Medicine, Osaka, Japan; ${ }^{4}$ Department of Pathology, School of Allied Health Science, Osaka University Faculty of Medicine, Osaka, Japan; ${ }^{5}$ Department of

Pathology, Wakayama Medical School, Wakayama, Japan

Polo-like kinase I (PLKI) is one of the serine threonine kinases that contributes to cell mitosis and is regarded as a marker of cellular proliferation. However, its protein expression in human carcinoma has not been studied in depth. We investigated PLKI expression in various thyroid neoplasms in order to elucidate its physiological significance in thyroid carcinoma. Normal follicular cells only occasionally expressed PLKI. In follicular tumours and anaplastic carcinoma, PLKI overexpression was not a common event and only 5.9\% of follicular adenoma, 7.1\% of follicular carcinoma, and $11.8 \%$ of anaplastic carcinoma overexpressed this protein. However, 43.7\% of papillary carcinoma overexpressed PLKI. Polo-like kinase I overexpression was more frequently observed in smaller papillary carcinoma lesions, and 62.5\% of microcarcinoma (ranging from $4 \mathrm{~mm}$ to $1.0 \mathrm{~cm}$ ) and even $66.7 \%$ of incidental carcinoma (less than $4 \mathrm{~mm}$ ) overexpressed it, whereas this phenomenon could only be seen in $20.0 \%$ of lesions larger than $4.0 \mathrm{~cm}$. Furthermore, PLKI overexpression was not related to cell-proliferating activity evaluated by Ki-67 labelling index, but it was inversely linked to UICC stage, extrathyroidal invasion, and the presence of poorly differentiated lesion as proposed by Sakamoto et al. These findings strongly suggest that, unlike other carcinomas previously studied, PLKI does not act as a cell cycle regulator but plays a constitutive role in papillary carcinoma especially in the early phase, and may contribute to the malignant transformation of this carcinoma. British Journal of Cancer (2004) 90, 4I4-4I8. doi:I0.1038/sj.bjc.660I540 www.bjcancer.com (c) 2004 Cancer Research UK

Keywords: PLKI; immunohistochemistry; thyroid

Thyroid carcinoma is one of the most common malignancies originating in the endocrine glands. There are two prominent histological types of thyroid carcinoma originating from follicular cells, papillary carcinoma and follicular carcinoma, which is comparatively rare. Follicular carcinoma is thought to arise from pre-existing follicular adenoma, although this has not yet been confirmed. The precursor lesion of papillary carcinoma has not yet been identified (Fagin, 2000). Generally, the biological activity of papillary carcinomas is mild, but when these lesions dedifferentiate and turn to anaplastic carcinoma (undifferentiated carcinoma), they grow rapidly and patients usually have a dire prognosis despite various therapeutic strategies (Aldinger et al, 1978). These polarised characteristics are typical of thyroid carcinoma, prompting many researchers to investigate factors linked to or triggering dedifferentiation. From a morphological perspective, Sakamoto et al (1983)found that papillary or follicular carcinoma with typical growth patterns, such as a solid, trabecular, or scirrhous pattern, shows a poorer prognosis than that with a pure papillary or follicular pattern only. Therefore, they hypothesised that these carcinomas are dedifferentiating, and designated such lesions as

*Correspondence: Y Ito; E-mail: ito0 I@kuma-h.or.jp

Received 23 June 2003; revised 3 November 2003; accepted 5 November 2003 poorly differentiated carcinoma, although this hypothesis has not been confirmed.

For the development of neoplasm, the activity of cell cycle progression, including mitosis, is important. To date, expression of various regulators of this event has been investigated in human neoplasms, including thyroid carcinoma. Polo-like kinase 1 (PLK1) is a serine threonine kinase, which belongs to the polo-like kinases (PLKs) family homologous to the Drosophila polo kinase (Golsteyn et al, 1994; Hamanaka et al, 1994). This protein is closely associated with cell cycle regulation. Golsteyn et al (1995) showed that, although the level of PLK1 protein expression is low in the G1 phase, it elevates in the S phase following maximal level in the G2/M phase. They also proved that its activity is also elevated during cell mitosis. Using cell lines and human carcinoma tissues, another group also demonstrated that PLK1 is a marker of cell proliferation, which could be useful for carcinoma diagnosis and may be a target for cancer chemotherapy (Holtrich et al, 1994; Yuan et al, 1997). To date, however, PLK mRNA expression has been investigated for a large number of human carcinoma specimens of only a few origins (Wolf et al, 1997; Knecht et al, 1999; Tokumitsu et al, 1999). In addition, PLK1 protein expression has been studied only for colorectum carcinoma (Takahashi et al, 2003), indicating that our knowledge of PLK1 expression and its physiological role in human carcinoma remains limited. 
These results prompted us to investigate PLK1 expression in thyroid neoplasms, to elucidate whether this kinase is associated with progression of this carcinoma.

\section{MATERIALS AND METHODS}

\section{Cell line}

A thyroid tumour cell line, NPA (papillary carcinoma), was established by our co-workers. Cells were cultured in Dulbecco's modified Eagle's medium (Nihonseiyaku, Tokyo, Japan) supplemented with $10 \%$ fetal calf serum (Dainippon Pharmaceutical, Tokyo, Japan), $500 \mathrm{U} \mathrm{ml}^{-1}$ penicillin (Gibco BRL, MD, USA), and $500 \mu \mathrm{g} \mathrm{ml}^{-1}$ streptomycin.

\section{Tissue specimens}

Tissue specimens of thyroid neoplasms were obtained from 149 patients who underwent surgery in the Department of Surgery, Kuma Hospital. This project was approved by the ethics committee of the hospital and informed consent was obtained from the participating patients. Our series involved 17 anaplastic (undifferentiated) carcinomas. These patients underwent surgery between 1987 and 2002. Between 1999 and 2001, 1205 patients underwent surgery for papillary or follicular carcinoma, and 155 (12.9\%) had lesions with solid, trabecular, or scirrhous growth patterns diagnosed as poorly differentiated carcinoma, as proposed by Sakamoto et al (1983). From these patients, we selected 87 papillary carcinomas and 28 follicular carcinomas (eight widely invasive and 20 minimally invasive carcinomas) for the present study. Of these cases, 28 papillary carcinomas and three follicular carcinomas were diagnosed as including poorly differentiated lesions according to Sakamoto et al (1983). Among this series of papillary carcinomas, 36 cases were diagnosed as microcarcinoma according to the WHO classification, because the maximal diameters were $1.0 \mathrm{~cm}$ or less (Hedinger et al, 1988). Generally, ultrasonography can detect tumours measuring $4 \mathrm{~mm}$ or more. In all, 24 of 36 cases were preoperatively diagnosed and surgical treatment was performed for the disease. However, the remaining 12 cases had not been detected by ultrasonography and were incidentally found in specimens following surgery for other thyroid diseases such as follicular adenoma, adenomatous goiter, and diffuse hyperplasia. In these cases, the maximal diameters were $3 \mathrm{~mm}$ or less. In addition, we selected 17 follicular adenomas from patients (Hedinger et al, 1988) who underwent surgery in 2002. For immunohistochemical study, tissues were fixed in $10 \%$ formalin and paraffin-embedded. For Western blot analysis, tissues were snap frozen in liquid nitrogen and stored at $-80^{\circ} \mathrm{C}$ until used.

\section{Antibody}

A mouse monoclonal anti-human PLK1 antibody and anti-Ki-67 antibody were obtained from Transduction Laboratories (Lexington, KY, USA) and Ylem (Rome, Italy), respectively. These were applied at concentrations of $1: 100$ and $1: 50$, respectively.

\section{Western blot analysis}

For Western blotting of PLK1, $20 \mu \mathrm{g}$ of proteins extracted from tissue specimens was electrophoresed on an $8 \%$ polyacrylamide gel. After blotting onto a PVDF membrane, the membrane filter was incubated with 1/1000 diluted anti-human PLK antibody at a concentration of $1: 200$ for $2 \mathrm{~h}$ at room temperature. The filter was washed three times with TBS containing $0.05 \%$ Tween 20 for $10 \mathrm{~min}$ each and then incubated with TBS containing $1 / 2500$ diluted peroxidase-conjugated goat antibody to mouse IgG (Promega, USA) for $1 \mathrm{~h}$. After washing the membrane three times with TBS containing $0.05 \%$ Tween 20 for $10 \mathrm{~min}$ each, it was developed by an enhanced chemiluminescence system (ECL, Amersham) according to the manufacturer's protocol.

\section{Immunohistochemistry}

Tissue sections $4 \mu \mathrm{m}$ thick were dewaxed and endogenous peroxidase activity was blocked with $0.3 \%$ hydrogen peroxide in methanol for $15 \mathrm{~min}$. For antigen retrieval, sections were immersed in $0.03 \mathrm{moll}^{-1}$ citrate buffer and incubated at $95^{\circ} \mathrm{C}$ for $40 \mathrm{~min}$. After rinsing in phosphate-buffered saline pH 7.2 (PBS), $10 \%$ bovine serum (Wako, Osaka, Japan) was applied for $20 \mathrm{~min}$ to block nonspecific reactions. Then, sections were incubated with a primary antibody at $4{ }^{\circ} \mathrm{C}$ overnight. After rinsing in PBS, specimens were treated with peroxidase-labelled anti-mouse and anti-rabbit immunoglobulins (Nichirei, Tokyo, Japan) for $30 \mathrm{~min}$. The peroxidase reaction was visualised by incubating the sections with $0.02 \% 3,3^{\prime}$-diaminobenzidine tetrahydrochloride in $0.05 \mathrm{M}$ Tris buffer with $0.01 \%$ hydrogen peroxide (Nichirei, Tokyo, Japan). The sections were counterstained with haematoxylin. Sections for the negative control were prepared using mouse immunoglobulin instead of primary antibody.

\section{Immunohistochemical evaluation}

Polo-like kinase 1 immunoreactivity was observed predominantly in the cytoplasm, and we regarded cells positive for PLK1 only when the immunoreactivity was clearly observed. Based on the positive tumour cell rate, we categorised all cases into four groups: $(-), 0-5 \%$; $(+), 6-29 \% ;(++), 30-59 \%$; and $(+++)$, more than $60 \%$. Cases graded as $(++)$ or $(+++)$ were regarded as highly overexpressing PLK1, whereas those graded as $(-)$ or $(+)$ were judged as showing low expression of PLK1. This categorisation is fundamentally similar to that in a previous immunohistochemical study using the same antibody (Takahashi et al, 2003).

We counted cells positive for Ki-67 by evaluating at least 300 cells in at least three randomly selected fields and calculated the labelling index (LI) for Ki-67. In papillary carcinoma, cases showing Ki-67 LI larger than 3\% were classified as the high group.

\section{Statistical analyses}

Fisher's exact probability test was adopted to examine the relationship between variables. A $P$-value less than 0.05 was considered significant.

\section{RESULTS}

Western blot analysis for PLK1 in thyroid tissues and cell lines

We investigated the expression of PLK1 in thyroid carcinomas and cell lines using Western blot analysis (Figure 1). Lanes 1-5 indicated papillary carcinoma, and lanes 2,3 , and 5 were positive for PLK1. Lanes 6-10 were follicular carcinomas, and lane 6 was the only positive case of PLK. There were no signals observed in normal thyroid tissues (lanes 11-14, Figure 1). Lanes 15-17 indicated anaplastic carcinomas, and a signal for PLK1 as a single band at $67 \mathrm{kDa}$ was observed in one case in lane 17. Polo-like kinase 1 signal was found in a thyroid carcinoma cell line, NPA (lane 18). These results for tissue specimens were coincident with our immunohistochemical findings in each case.

\section{Immunohistochemistry for PLK1 in thyroid neoplasms}

Polo-like kinase 1 was only occasionally expressed in normal follicular cells, as well as infiltrating lymphocytes (Figure 2A). 


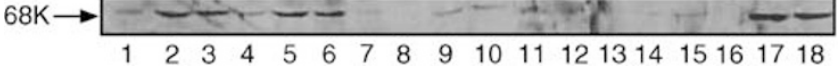

Figure I Western blot of PLKI on thyroid carcinoma tissues and thyroid tumour cell lines. Twenty $\mu$ g of total cellular proteins were electrophoresed on 12\% SDS-PAGE and then Western blot of PLKI was performed as described in Materials and Methods. Lanes $\mathrm{I}-5$ indicate papillary carcinoma, lanes 6- 10 follicular carcinoma, lanes II - I4 normal thyroid tissue, lanes 15-17 anaplastic carcinoma, and lane 18 thyroid carcinoma cell line, NPA.
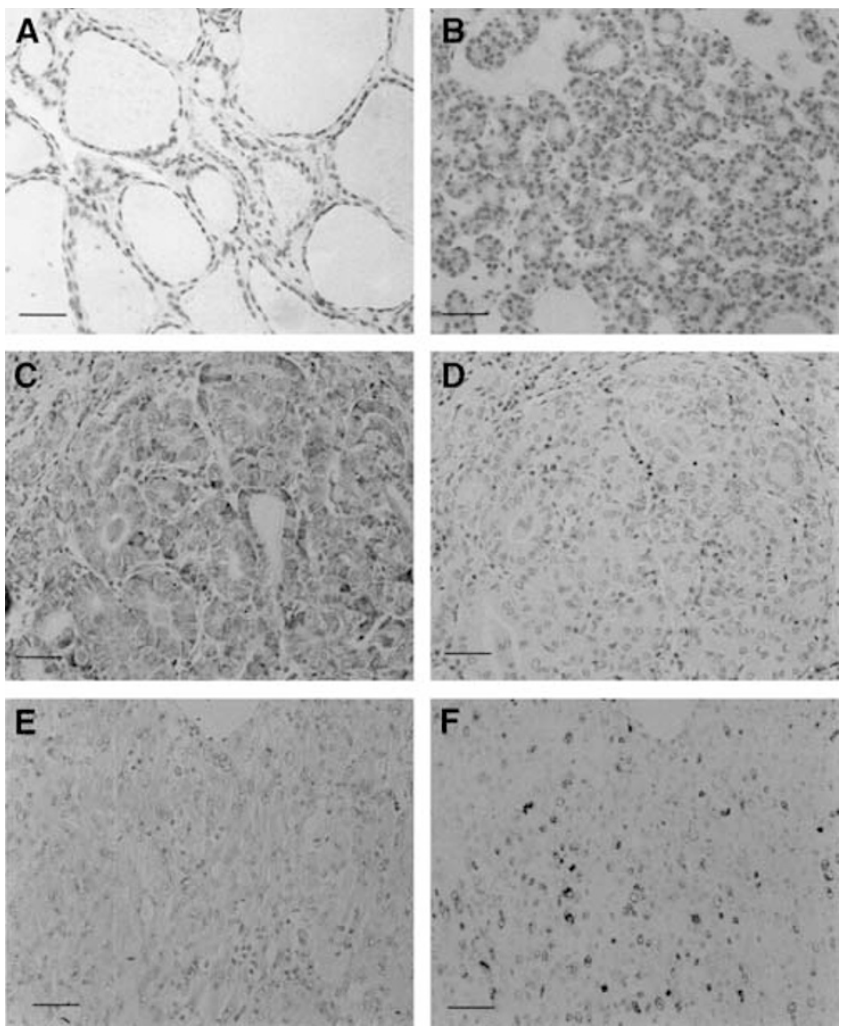

Figure 2 Immunostaining of PLKI and Ki-67 in thyroid tissues. (A) PLKI was not expressed in normal follicules. (B) Absence of PLKI expression in follicular carcinoma. (C) PLKI overexpression in papillary carcinoma. (D) Absence of Ki-67 antigen expression in a serial section with (C). (E) PLKI was not overexpressed in anaplastic carcinoma. (F) Ki-67 antigen expression is frequently observed in a serial section with (E) Scale bar $150 \mu \mathrm{m}$.

Polo-like kinase 1 expression in various types of thyroid neoplasm is summarised in Table 1.

\section{Follicular carcinoma and adenoma}

In follicular tumours, PLK1 overexpression was not frequently observed. Only one of the 17 adenomas (5.9\%) and two of the 28 carcinomas $(7.1 \%)$ were classified in the high group (Figure $2 \mathrm{~B}$ ). There was no significant difference in the incidence of PLK1 overexpression between follicular adenoma and carcinoma.

\section{Papillary carcinoma}

Papillary carcinoma overexpressed PLK1 more frequently than follicular carcinoma $(P=0.0002)$, and 38 cases were judged in the high group (43.7\%) (Figure 2C). Among the papillary carcinomas in this study, 36 cases were microcarcinoma with maximal diameters of $1.0 \mathrm{~cm}$ or less. Of these lesions, 24 were preoperatively diagnosed and their sizes ranged from $4 \mathrm{~mm}$ to $1.0 \mathrm{~cm}$. Polo-like
Table I Expression of PLKI in thyroid neoplasms

\begin{tabular}{|c|c|c|c|c|c|}
\hline \multirow[b]{3}{*}{ Histological type } & \multicolumn{5}{|c|}{ PLKI expression } \\
\hline & \multicolumn{2}{|c|}{ Low } & \multicolumn{2}{|c|}{ High } & \multirow[b]{2}{*}{ Total } \\
\hline & - & + & ++ & ++ & \\
\hline Anaplastic caccinoma* & 7 & 8 & 0 & 2 & 17 \\
\hline Papillary carcinoma**** & 26 & 23 & 27 & 11 & 87 \\
\hline Follicular carcinoma*** & 21 & 5 & 2 & 0 & 28 \\
\hline Follicular adenoma & 14 & 2 & 1 & 0 & 17 \\
\hline
\end{tabular}

* $P=0.0143$, ** $P=0.0002$.

Table 2 Relationship between PLKI expression in papillary carcinoma and tumour size

\begin{tabular}{|c|c|c|c|c|c|}
\hline & \multicolumn{5}{|c|}{ PLKI expression } \\
\hline & \multicolumn{2}{|c|}{ Low } & \multicolumn{2}{|c|}{ High } & \multirow[b]{2}{*}{ Total } \\
\hline & - & + & ++ & ++ & \\
\hline$\leqslant 0.3 \mathrm{~cm}^{\mathrm{a}, \mathrm{b}}$ & 2 & 2 & 6 & 2 & 12 \\
\hline$>0.3 \mathrm{~cm}, \leqslant 1.0 \mathrm{~cm}^{b}$ & 8 & I & | | & 4 & 24 \\
\hline$>1.0 \mathrm{~cm},<4.0 \mathrm{~cm}^{c}$ & 9 & 15 & 10 & 2 & 36 \\
\hline$>4.0 \mathrm{~cm}^{c}$ & 7 & 5 & 0 & 3 & 15 \\
\hline Total & 26 & 23 & 27 & | | & 87 \\
\hline
\end{tabular}

aThese carcinomas were incidentally found in surgical specimens resected for other thyroid diseases (incidental carcinoma). b vs c: $P=0.0020$.

kinase 1 overexpression was observed in 15 of these cases (62.5\%). Furthermore, the remaining 12 cases were incidental carcinomas, $3 \mathrm{~mm}$ in diameter or smaller. Also in these cases, PLK1 was frequently overexpressed and eight cases $(66.7 \%)$ were regarded in the high group. As shown in Table 2, larger papillary carcinomas showed a decrease in PLK1 expression $(P=0.0020)$, and of 15 tumours larger than $4.0 \mathrm{~cm}$, only three $(20.0 \%)$ overexpressed PLK1.

We investigated the relationship between PLK1 overexpression and other clinicopathological parameters of papillary carcinoma (Table 3). For this analysis, incidental carcinomas were omitted, because most parameters for these cases were unknown. Polo-like kinase overexpression was inversely linked to UICC early stage $(P=0.0093)$, extrathyroidal invasion $(P=0.0372)$, and the presence of a lesion showing solid, trabecular, or scirrhous growth pattern $(P=0.0005)$. Furthermore, we compared PLK1 expression and $\mathrm{Ki}-67 \mathrm{LI}$ in papillary carcinoma. Generally, cell-proliferating activity of thyroid carcinoma except for anaplastic carcinoma is very low. Also in papillary carcinoma in our series, when we set the cutoff value at $3 \%$, only 17 cases were classified in the high group. As shown in Figure 2C and D, coexpression of PLK1 and Ki-67 was only occasionally seen, and we could not establish any relationship between PLK1 expression and Ki-67 LI in papillary carcinoma (Table 3).

\section{Anaplastic carcinoma}

Anaplastic carcinoma originats from papillary and follicular carcinomas, and has a high activity of development. Indeed, in our series, Ki-67 LI was high and averaged $38.7+16.6(15.3-60.9)$. However, in this carcinoma, PLK1 overexpression was seen only in two of the 17 cases (11.8\%) (Figure 2E), and the incidence was significantly lower than that in papillary carcinoma $(P=0.0143)$. Furthermore, we could not find any relationship between PLK1 expression and Ki-67 antigen expression in this carcinoma (Figure $2 \mathrm{E}$ and $\mathrm{F})$. 
Table 3 Relationship between PLKI expression and clinicopathlogical parameters other than tumour size of papillary carcinoma excluding incidental carcinoma

\begin{tabular}{|c|c|c|c|}
\hline & Low & High & Total \\
\hline Age (years) (mean \pm s.d.) & $56.4 \pm 11.4$ & \multicolumn{2}{|c|}{$\begin{array}{r}33.3 \pm 14.0 \\
\text { NS }\end{array}$} \\
\hline \multicolumn{4}{|l|}{ Gender } \\
\hline Male & 5 & 3 & 8 \\
\hline Female & 40 & \multicolumn{2}{|c|}{ NS } \\
\hline \multicolumn{4}{|l|}{ Lymph node metastasis } \\
\hline Absent & 19 & 12 & 31 \\
\hline Present & 26 & 18 & 44 \\
\hline \multicolumn{4}{|l|}{ Multiple tumour formation } \\
\hline Absent & 24 & 20 & 44 \\
\hline Present & 21 & \multicolumn{2}{|c|}{ NS } \\
\hline \multicolumn{4}{|l|}{ UICC stage } \\
\hline । & 13 & 18 & 31 \\
\hline$\|$ & 14 & 5 & 19 \\
\hline \multirow[t]{2}{*}{ III or IV } & 18 & 7 & 25 \\
\hline & \multicolumn{3}{|c|}{$P=0.0093($ stage I vs stage $\geqslant \| 1)$} \\
\hline \multicolumn{4}{|l|}{ Extrathyroidal invasion } \\
\hline Absent & 29 & 26 & 55 \\
\hline Present & 16 & 4 & 20 \\
\hline \multicolumn{4}{|c|}{ Solid, scirrhous, or trabecular growth patterns } \\
\hline Absent & 21 & 26 & 47 \\
\hline Present & 24 & 4 & 28 \\
\hline \multicolumn{4}{|l|}{ Ki-67 LI } \\
\hline Low & 35 & 23 & 58 \\
\hline High & 10 & 7 & 17 \\
\hline & & \multicolumn{2}{|c|}{ NS } \\
\hline Total & 45 & 30 & 75 \\
\hline
\end{tabular}

\section{DISCUSSION}

In this study, we demonstrated that (1) follicular cells in normal follicles only occasionally expressed PLK1, (2) the incidence of PLK1 overexpression was not high in either follicular tumours or anaplastic carcinoma, and (3) in papillary carcinoma, PLK1 overexpression was more frequently observed in cases showing low biologically aggressive phenotypes, although it was not related to cell-proliferating activity.

To date, PLK mRNA expression has been studied in a few types of carcinoma such as non-small cell lung carcinoma, squamous cell carcinoma of the head and neck, gastric carcinoma, and oesophageal carcinoma (Wolf et al, 1997; Knecht et al, 1999; Tokumitsu et al, 1999). Except for that in gastric carcinoma, the PLK mRNA level in these carcinomas was directly linked to the biologically aggressive phenotype of the tumour and/or worse prognosis for the patient (Wolf et al, 1997; Knecht et al, 1999; Tokumitsu et al, 1999). Furthermore, Takahashi et al (2003) demonstrated that, in human colorectal carcinoma, PLK1 protein overexpression was directly related to the proliferating cell nuclear antigen (PCNA) LI, as well as some other parameters reflecting the biological aggressiveness of the tumour such as Dukes' classification, primary tumour invasion, lymph node metastasis, and aberrant p53 expression. These results are not discrepant with and even supported by those obtained from previous in vitro studies that PLK1 plays a significant role in cellproliferating activity (Holtrich et al, 1994; Golsteyn et al, 1995; Yuan et al, 1997).

However, our findings are sharply in contrast with those in other carcinomas, and the physiological significance of PLK1 in thyroid tumours seems complicated. A high incidence of PLK1 overexpression was observed in papillary carcinoma. As previously reported, cell-proliferating activity of papillary carcinoma is generally low (Erickson et al, 1998), and in our series, Ki-67 LI was less than $3 \%$ in most cases. We investigated the relationship between PLK1 overexpression and cell-proliferating activity of papillary carcinoma, but there was no association detected, indicating that PLK1 does not play a role in regulating cell proliferation. Instead, we found that PLK1 is more frequently overexpressed in papillary carcinoma with low biological aggressiveness, because its expression was inversely linked to certain clinicopathological parameters such as tumour size, UICC stage, extrathyroidal invasion, and the presence of poorly differentiated lesion. Especially, it is interesting to that PLK1 is more frequently overexpressed in papillary microcarcinoma and even in incidental carcinoma, which is smaller than $4 \mathrm{~mm}$ in maximal diameter. Recently, we performed a trial of observation without surgical treatment for papillary microcarcinoma patients, and found that most of these tumours do not enlarge, indicating that these lesions do not grow or grow only slowly (Ito et al, 2003). These findings strongly suggest that PLK1 is not related to growth of papillary carcinoma. Another interesting finding is the frequent lack of PLK1 expression in anaplastic carcinoma. Anaplastic carcinoma is known to arise from papillary and follicular carcinomas and has high mitotic activity as demonstrated by Erickson et al (1998) as well as this study. However, in our series, PLK1 overexpression was observed only in $11.8 \%$ of cases. According to these findings, we can conclude that PLK1 is not likely to contribute directly to the mitosis of papillary carcinoma cells. This means that the physiological significance of PLK1 in thyroid papillary carcinoma is unique and completely different from that in all other carcinomas studied to date. One explanation of these results is that PLK1 plays an oncogenic role and is constitutively required in papillary carcinoma during the early phase, while it is less necessary for the development of this carcinoma during the advanced stage. Although the mechanism of malignant transformation from normal follicle to papillary carcinoma remains to be elucidated, our finding that PLK1 is frequently overexpressed in incidental carcinomas suggests that PLK1 expression may even play a role in the carcinogenesis of papillary carcinoma.

In follicular tumours, PLK1 overexpression was not frequently detected, and there was no significant difference in the incidence between follicular carcinoma and follicular adenoma. It is therefore suggested that this protein is not likely to play a role in the progression of this tumour or in the transformation of follicular adenoma to carcinoma.

In summary, the findings of this study strongly suggest that PLK1 plays a constitutive role in thyroid papillary carcinoma especially during the early phase, and may be related to the malignant transformation of this lesion. Further studies are necessary to elucidate the detailed mechanism by which this protein contributes to carcinogenesis and the development of this carcinoma.

\section{REFERENCES}

Aldinger KA, Samaan NA, Ibanez M, Hill Jr CS (1978) Anaplastic carcinoma of the thyroid: a review of 84 cases of spindle and giant-cell carcinoma of the thyroid. Cancer 41: 2267-2275
Erickson LA, Jin L, Wollan PC, Thompson GB, van Heerden J, Lloyd RV (1998) Expression of p27Kip1 and Ki-67 in benign and malignant thyroid tumors. Mod Pathol 11: 169-174 
Fagin JA (2000) Molecular genetics of tumors of thyroid follicular cells. In The Thyroid, Braverman LE, Utiger RD (eds) 8th edn, pp 886-898. Lippincott Williams \& Wilkins: Philadelphia

Golsteyn RM, Schultz SJ, Bartek J, Ziemiecki A, Ried T, Nigg EA (1994) Cell cycle analysis and chromosomal localization of human Plk1, a putative homologue of the mitotic kinases Drosophila polo and Saccharomyces cerevisiae Cdc5. J Cell Sci 107: 1509-1517

Golsteyn RM, Schulttz SJ, Bartek J, Ziemiecki A, Ried T, Nigg EA (1995) Cell cycle regulation of the activity and subcellular localization of PLK1, a human protein kinase implicated in mitotic spindle function. J Cel Biol 129: $1617-1628$

Hamanaka R, Maloid S, Smith MR, O'Connell CD, Longo DL, Ferris DK (1994) Cloning and characterization of human and murine homologues of the Drosophila polo serine-threonine kinase. Cell Growth Differ 5: 249-257

Hedinger C, Williams ED, Sobin LH (1988) Histological typing of thyroid tumours. 2nd edn, pp 1-66 (World Health Organization) New York: Springer-Verlag

Holtrich U, Wolf G, Brauninger A, Karn T, Bohme B, Rubsamen-Waigmann $\mathrm{H}$, Strebhardt K (1994) Induction and down-regulation of PLK, a human serine/threonine kinase expressed in proliferating cells and tumors. Proc Natl Acad Sci USA 91: 1736-1740

Ito Y, Uruno R, Nakano K, Takamura Y, Miya A, Kobayashi K, Yokozawa T, Matsuzuuka F, Kuma S, Kuma K, Miyauchi A (2003) An observation trial without surgical treatment in patients with papillary microcarcinoma of the thyroid. Thyroid 13: $381-388$

Knecht R, Elez R, Oechler M, Solbach C, von Illberg C, Strebhardt K (1999) Prognostic significance of polo-like kinase (PLK) expression in squamous cell carcinomas of the head and neck. Cancer Res 59: $2794-2797$

Sakamoto A, Kasai N, Sugano H (1983) Poorly differentiated carcinoma of the thyroid. A clinicopathological entity for a high-risk group of papillary and follicular carcinomas. Cancer 52: $1849-1855$

Takahashi T, Sano B, Nagata T, Kato H, Sugiyama Y, Kunieda K, Kimura M, Okano Y, Saji S (2003) Polo-like kinase 1(PLK1) is overexpressed in primary colorectal cancers. Cancer Sci 94: $148-152$

Tokumitsu Y, Mori M, Tanaka S, Akazawa K, Nakano S, Niho Y (1999) Prognostic significance of polo-like kinase expression in esophageal carcinoma. Int J Oncol 15: 687-692

Wolf G, Elez R, Doermer A, Holtrich U, Ackermann H, Stutte HJ, Altmannsberger HM, Rubsamen-Waigmann H, Strebhardt K (1997) Prognostic significance of polo-like kinase (PLK) expression in nonsmall cell lung cancer. Oncogene 14: 543-549

Yuan J, Horlin A, Hock B, Stutte HJ, Rubsamen-Waigmann H, Strebhardt K (1997) Polo-like kinase, a novel marker for cellular proliferation. Am J Pathol 150: $1165-1172$ 